\title{
Enhanced Ex Vivo Generation of Erythroid Cells from Human Induced Pluripotent Stem Cells in a Simplified Cell Culture System with Low Cytokine Support
}

\author{
Claudia Bernecker, Mania Ackermann, ${ }^{2,3}$ Nico Lachmann, ${ }^{2,3}$ Lisa Rohrhofer, ${ }^{1}$ Holm Zaehres, ${ }^{4}$ \\ Marcos J. Araúzo-Bravo, ${ }^{5,6}$ Emile van den Akker, ${ }^{7}$ Peter Schlenke, and Isabel Dorn ${ }^{1}$
}

Red blood cell (RBC) differentiation from human induced pluripotent stem cells (hiPSCs) offers great potential for developmental studies and innovative therapies. However, ex vivo erythropoiesis from hiPSCs is currently limited by low efficiency and unphysiological conditions of common culture systems. Especially, the absence of a physiological niche may impair cell growth and lineage-specific differentiation. We here describe a simplified, xeno- and feeder-free culture system for prolonged RBC generation that uses low numbers of supporting cytokines [stem cell factor (SCF), erythropoietin (EPO), and interleukin 3 (IL-3)] and is based on the intermediate development of a "hematopoietic cell forming complex (HCFC)." From this HCFC, CD43 ${ }^{+}$hematopoietic cells (purity $>95 \%$ ) were continuously released into the supernatant and could be collected repeatedly over a period of 6 weeks for further erythroid differentiation. The released cells were mainly CD $34^{+} / \mathrm{CD}_{4} 5^{+}$ progenitors with high erythroid colony-forming potential and $\mathrm{CD} 36^{+}$erythroid precursors. A total of $1.5 \times 10^{7}$ cells could be harvested from the supernatant of one six-well plate, showing 100- to 1000-fold amplification during subsequent homogeneous differentiation into $\mathrm{GPA}^{+}$erythroid cells. Mean enucleation rates near 40\% (up to $60 \%$ ) further confirmed the potency of the system. These benefits may be explained by the generation of a niche within the HCFC that mimics the spatiotemporal signaling of the physiological microenvironment in which erythropoiesis occurs. Compared to other protocols, this method provides lower complexity, less cytokine and medium consumption, higher cellular output, and better enucleation. In addition, slight modifications in cytokine addition shift the system toward continuous generation of granulocytes and macrophages.

Keywords: induced pluripotent stem cells, hematopoiesis, erythropoiesis, niche, red blood cell

\section{Introduction}

$\mathrm{T}$ HE EX VIVO MANUFACTURING of red blood cells (RBCs) from human induced pluripotent stem cells (hiPSCs) holds great promise for the development of innovative therapeutic and diagnostic strategies. In the future, cultured $\mathrm{RBCs}$ (cRBCs) may serve as RBC products for use in severely immunized patients, antibody screening tools, disease model systems, or tools for developmental studies. However, despite some progress over the past few years, RBC generation from hiPSCs is still limited by low expansion rates, a lack of adult hemoglobin expression, and insufficient enucleation $(<20 \%)$ [1-3]. In this context, mimicking erythropoiesis during the time course of early human development remains a challenge. To overcome a lack of understanding of the molecular mechanisms that occur during embryogenesis, complex and unphysiological culture conditions with high amounts of sometimes more than 10 different cytokines are

\footnotetext{
${ }^{1}$ Department of Blood Group Serology and Transfusion Medicine, Medical University Graz, Graz, Austria.

${ }^{2}$ RG Translational Hematology of Congenital Diseases, Institute of Experimental Hematology, Hannover Medical School, Hannover, Germany.

${ }^{3}$ REBIRTH Cluster of Excellence, Hannover Medical School, Hannover, Germany.

${ }_{5}^{4}$ Department of Anatomy and Molecular Embryology, Ruhr-University Bochum, Bochum, Germany.

${ }^{5}$ Computational Biology and Systems Biomedicine Research Group, Biodonostia Health Research Institute, San Sebastián, Spain.

${ }^{6}$ IKERBASQUE, Basque Foundation for Science, Bilbao, Spain.

${ }^{7}$ Department of Hematopoiesis, Sanquin Research, Amsterdam, the Netherlands.

(C) Claudia Bernecker et al. 2019; Published by Mary Ann Liebert, Inc. This Open Access article is distributed under the terms of the Creative Commons Attribution Noncommercial License (http://creativecommons.org/licenses/by-nc/4.0/) which permits any noncommercial use, distribution, and reproduction in any medium, provided the original author(s) and the source are cited.
} 
used. Ex vivo erythropoiesis models are further biased by the absence of a microenvironmental niche, hindering a biomimetic recapitulation of the multistep physiological maturation process.

Hematopoietic cells arise in overlapping waves. A transient wave of primitive hematopoiesis occurs in the yolk sac and is responsible for the blood supply of the early embryo. Primitive erythroblasts express the embryonic globin genes Gower I $\left(\zeta_{2} \varepsilon_{2}\right)$ and Gower II $\left(\alpha_{2} \varepsilon_{2}\right)$ and are able to enucleate in the circulation [4,5]. In the second wave, erythroidmyeloid progenitors appear in the yolk sac. They migrate to the fetal liver and produce definitive erythroblasts, which express primarily fetal hemoglobin [6,7]. With the emergence of hematopoietic stem cells (HSCs) in the aortagonad-mesonephros (AGM) region, this transient system is replaced by a third wave of lifelong definitive hematopoiesis that switches after birth from the fetal liver to the bone marrow (BM). Definitive RBCs derived from HSCs in the $\mathrm{BM}$ express mainly adult globin genes $\left(\alpha_{2} \beta_{2}\right)$ [7-9].

Hematopoietic and erythroid fate are orchestrated by a complex network of different cell types, humoral factors, and extracellular matrix molecules, which collectively compose a physiological cell type-specific niche $[10,11]$. Due to ethical concerns and the inaccessibility of human embryos, the composition and spatiotemporal transformation of this niche during embryonic development remain largely unknown.

Since the pioneering discovery that somatic cells can be reprogrammed for pluripotency, several culture systems for the ex vivo generation of RBCs from hiPSCs have been established. Although they differ from each other in their experimental setups, the protocols share a common strategy for inducing erythropoiesis. These methods consist of different culture phases intended to induce mesodermal and hematopoietic commitment followed by the induction of erythropoiesis, the amplification of erythroid precursor cells, and finally the maturation of precursors into enucleated RBCs. For initial mesodermal and hematopoietic induction, two major technical approaches exist: (1) coculture of hiPSCs on human- or animal-derived stroma cells [12-16] and (2) culture of hiPSCs in suspension to form aggregates, termed embryoid bodies (EBs), which contain derivates of all three germ layers [17-20].

The majority of established protocols exhibit disadvantages in that they are very complex (with 3-9 different phases), time consuming, expensive, and unphysiological due to extensive cytokine support (up to 13 different growth factors). Furthermore, in most protocols, the hematopoietic cells undergo one or more digestion and purification steps, further increasing the complexity of the process and destroying potentially necessary cell interactions in the artificial niche.

Our group recently reported the manufacturing of cRBCs from hiPSC lines of different origins using an EB-based suspension system [17]. Consistent with reports from other groups, we observed robust and homogeneous erythroid differentiation accompanied by low amplification and limited enucleation $(\leq 25 \%)[12,14,15,18]$. One reason for the insufficient expansion in established systems might be the bypass of a highly proliferative erythroid progenitor stage under ex vivo conditions. In this context, only a few cells with characteristics of HSCs $\left(\mathrm{CD} 34^{+} / \mathrm{CD} 45^{+}\right)$or erythroid progenitors $\left(\mathrm{CD} 36^{+} / \mathrm{CD} 45^{+}\right)$have been generated.
More recently, we developed a simple, but robust protocol for the prolonged generation of granulocytes and macrophages from hiPSCs with minimal handling time and low cytokine support [21]. Based on these observations, we here created an improved model for prolonged ex vivo RBC generation from hiPSCs. In this system, an intermediate "hematopoietic cell forming complex (HCFC)" is established from which immature hematopoietic progenitors and erythroid precursors emerge continuously over 6 weeks. During further erythroid differentiation, cells exhibit increased proliferation and terminal maturation with enhanced enucleation rates for hiPSC-derived RBCs (up to 60\%). Compared to other systems, this system is a simpler and less expensive alternative. The reduced cytokine support and the maintenance of intact three-dimensional (3D) organization during hematopoietic specification may roughly mimic the physiological niche. Hence, this model might be less artificial and more suitable for studies on human erythropoiesis than conventional systems.

\section{Materials and Methods}

\section{hiPSC lines}

hiPSC lines from cord blood CD34 ${ }^{+}$cells (CD34-iPSCs) [17] and CD36 ${ }^{+}$erythroblasts (PEB-iPSCs) were used as independent biological replicates. Human $\mathrm{CD}^{+} 6^{+}$basophilic erythroblasts were reprogrammed by lentiviral overexpression of OCT4, SOX2, KLF4, and c-MYC. The details are given in the Supplementary Materials and Methods in Supplementary Data. Written informed consent was obtained before sampling in accordance with the Declaration of Helsinki, and the study was approved by the local ethics committee (EK27-165ex 14/15; Medical University Graz, Graz, Austria).

\section{Hematopoietic induction}

Undifferentiated iPSCs were maintained on Matrigel ${ }^{\circledR}$ (Corning) with StemMACS iPS Brew XF (Miltenyi Biotec). For EB formation, colonies (>23 passages) were detached with collagenase IV. Cell clumps were seeded on low-binding suspension plates (Nunclon Sphera; Thermo Fisher Scientific) and cultivated for 5 days in human embryonic stem cell (hESC) medium without bFGF [17]. Thereafter, 10-15 spherical EBs were transferred to six-well tissue culture plates (Sarstedt) with STEMdiff ${ }^{\mathrm{TM}}$ APEL $^{\mathrm{TM}} 2$ Medium (STEM CELL Technologies), 5\% PFHM-II Protein-Free Hybridoma Medium (Thermo Fisher Scientific), $5 \mathrm{ng} / \mathrm{mL}$ interleukin 3 (IL-3; PeproTech), 100 ng/mL stem cell factor (SCF; PeproTech), and $3 \mathrm{U} / \mathrm{mL}$ erythropoietin (EPO; Erypo; Janssen Biologics B.V.). The medium was changed weekly (Fig. 1).

\section{Erythroid differentiation}

Single cells released into the supernatant were collected and cultured for 18 days in an established three-phase erythropoiesis assay [17]. Cells cultured in Iscove's liquid medium (Biochrom) containing 10\% human plasma (Octapharm), $10 \mu \mathrm{g} / \mathrm{mL}$ insulin (Sigma-Aldrich), and $330 \mu \mathrm{g} / \mathrm{mL}$ human holotransferrin (Spicac) were stimulated with $100 \mathrm{ng} /$ $\mathrm{mL} \mathrm{SCF}, 5 \mathrm{ng} / \mathrm{mL} \mathrm{IL}-3$, and $3 \mathrm{U} / \mathrm{mL}$ EPO from days 0 to 8 , with $100 \mathrm{ng} / \mathrm{mL} \mathrm{SCF}$ and $3 \mathrm{U} / \mathrm{mL}$ EPO from days 8 to 11 , 


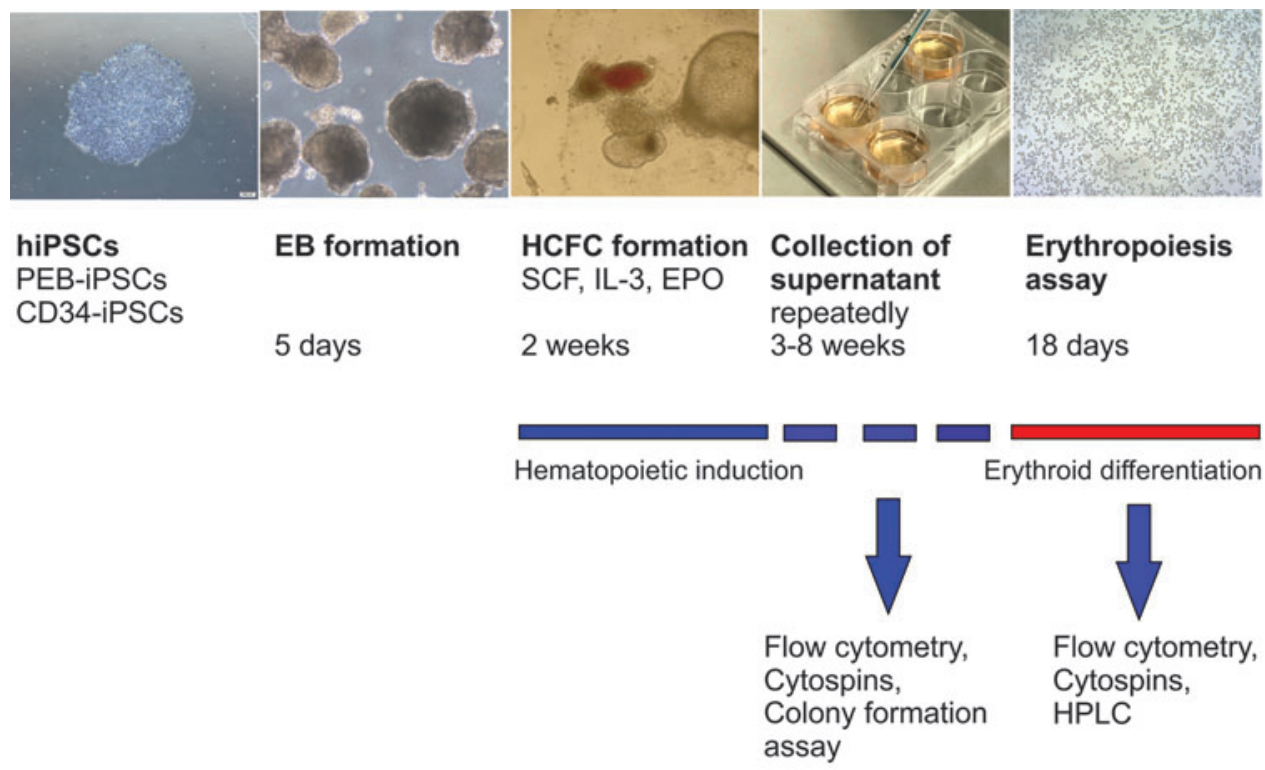

FIG. 1. Schematic illustration of the cell culture system. (1) Hematopoietic induction: colonies of undifferentiated hiPSCs were transferred into low-binding plates to induce EB formation. After 5 days, spherical EBs were further cultured on adherent plates in APEL ${ }^{\mathrm{TM}}$ medium containing SCF, EPO, and IL-3. The medium was changed weekly. Within 2 weeks, an HCFC was established, from which hematopoietic cells were continuously released into the supernatant and harvested for further characterization. (2) Erythroid differentiation: cells released into the supernatant were harvested and differentiated into RBCs in a three-phase erythropoiesis system over 18 days. Hematopoietic and erythroid differentiation were monitored by colony formation assay, flow cytometry, microscopy, and hemoglobin analysis. EB, embryoid body; EPO, erythropoietin; HCFC, hematopoietic cell forming complex; hiPSCs, human induced pluripotent stem cells; IL-3, interleukin 3; RBCs, red blood cells; SCF, stem cell factor.

and with $3 \mathrm{U} / \mathrm{mL}$ EPO from days 11 to 18 . Hematopoietic and erythroid differentiation were monitored by flow cytometry, colony formation in semisolid media, and microscopic evaluation (Supplementary Materials and Methods in Supplementary Data and Supplementary Table S1). The hemoglobin composition of the cRBCs after 15 days of ex vivo erythropoiesis was analyzed by high-performance cation exchange liquid chromatography on a Waters Alliance 2690 system [22].

\section{EB suspension model}

In a subset of experiments, hematopoietic induction of hiPSCs was additionally performed in our conventional EB suspension model, as previously described [17]. Therefore, EBs were stimulated for 20 days with a cocktail of eight cytokines and thereafter dissociated with collagenase. Single cells were further cultured in the three-phase erythropoiesis system. In a subset of experiments, CD $43^{+}$-sorted cells and unsorted cells from the same starting population were compared for further erythroid maturation.

\section{Characterization of the HCFC}

After removal of the supernatant, adherent cells of the HCFC were incubated with collagenase B (Roche Diagnostics) for further digestion. Detached cells were singularized by pipetting before staining with respective antibodies (Supplementary Table S1) for measurement on a Navios ${ }^{\mathrm{TM}}$ flow cytometer (Beckman Coulter). In addition, cells were sorted on a Becton Dickinson FACSAria IIu for analysis of
$\mathrm{CD} 43^{+} / \mathrm{CD} 31^{-}$and $\mathrm{CD} 31^{+} / \mathrm{CD} 43^{+}$subpopulations. Sorted populations were analyzed for their hematopoietic and endothelial potential by hematopoietic colony and net formation assays (No. ECM625; Chemicon) according to the manufacturer's instructions. As a positive control, human umbilical vein endothelial cells (HUVECs; PromoCell) were used.

\section{Statistics}

Statistical analyses were performed with IBM SPSS statistics 25 software using Mann-Whitney U tests for independent samples and Wilcoxon tests for paired samples.

\section{Results}

\section{Generation of iPSCs from human basophilic erythroblasts (PEB-iPSCs)}

Based on our observations of the continuous generation of macrophages and granulocytes from hiPSCs by an intermediate myeloid cell-forming complex, we aimed to develop a differentiation technique to generate erythrocytes continuously in an intact 3D network under low cytokine support. For this purpose, CD34-iPSCs [17] and PEB-iPSCs were used as independent biological replicates. Cells on day 7 of ex vivo erythropoiesis from adult CD34 ${ }^{+}$HSCs were used for generation of PEB-iPSCs (Supplementary Fig. S1). $\mathrm{CD} 6^{+}$basophilic erythroblasts (purity 98\%) were reprogrammed for pluripotency using a lentiviral vector expressing POU5F1/OCT4, SOX2, KLF4, and c-MYC as a polycistronic unit under the control of the retroviral SFFV 
promoter. After $\sim 25$ days, ESC-like colonies were selected based on their morphology for further propagation (Supplementary Fig. S1A). Immunofluorescence staining confirmed the expression of the pluripotency markers POU5F1/ OCT4, SOX2, SSEA4, TRA1-60, and TRA1-81, as well as the activity of alkaline phosphatase (Supplementary Fig. S1B). Karyotyping showed a normal karyotype without aberrations (Supplementary Fig. S1C). To demonstrate successful reprogramming for pluripotency, the PEB-iPSC global gene expression profile was compared to the H1-hESC expression profile. The canonical pluripotency factors POU5F1/OCT4, SOX2, LIN28, KLF4, and NANOG lined up on the diagonal in a pairwise scatter plot comparing PEB-iPSCs and H1-hESCs. It was found that $94.6 \%$ of the transcripts were similarly expressed (using a twofold threshold on a $\log _{2}$ scale) in the PEBiPSCs and H1-hESCs (Supplementary Fig. S1D).

\section{Hematopoietic induction of hiPSCs}

EB formation was induced to promote germ layer induction, and compact, spherical EBs were obtained after 5 days (Fig. 1). To induce further hematopoietic specification, EBs were allowed to adhere to the plastic surface and were stimulated with SCF, EPO, and IL-3. Medium exchange was performed only once a week. Under these conditions, EBs became increasingly blistered, and surrounding stromal layers were generated. Within 2 weeks, an adherent cellular complex, termed "hematopoietic cell forming complex (HCFC)," was established, consisting of blistered spheroids with "red islands," surrounded by a network of stromal cells. Nonadherent cells have died during this time period. Following this, from this HCFC, single cells were continuously released into the supernatant (Fig. 2A-C). The quantity of released cells increased from weeks 3 to 5 of cytokine stimulation and subsequently decreased until the culture was exhausted (week 8) (Fig. 2D, E). With regard to PEB-iPSCs $(n=5)$, as many as $2.4 \times 10^{6} \pm 0.35 \times 10^{6}$ cells (CD34-iPSCs: $2.2 \times 10^{6} \pm 1.2 \times 10^{6}$ cells, $n=5$ ) could be harvested from one well of a six-well plate during the entire HCFC culture period. This cell number arose out of $\sim 0.07 \times 10^{6}$ seeded iPSCs forming one $\mathrm{HCFC} /$ well. The formation of stromal layers and the shaping of vesicular spheroids during the first 2 weeks were crucial for the generation of the HCFC. Lack in $3 \mathrm{D}$ organization or stromal cell generation resulted in the absence of red islands and single cells in the supernatant (Supplementary Fig. S2). Since the appearance of dense, flat
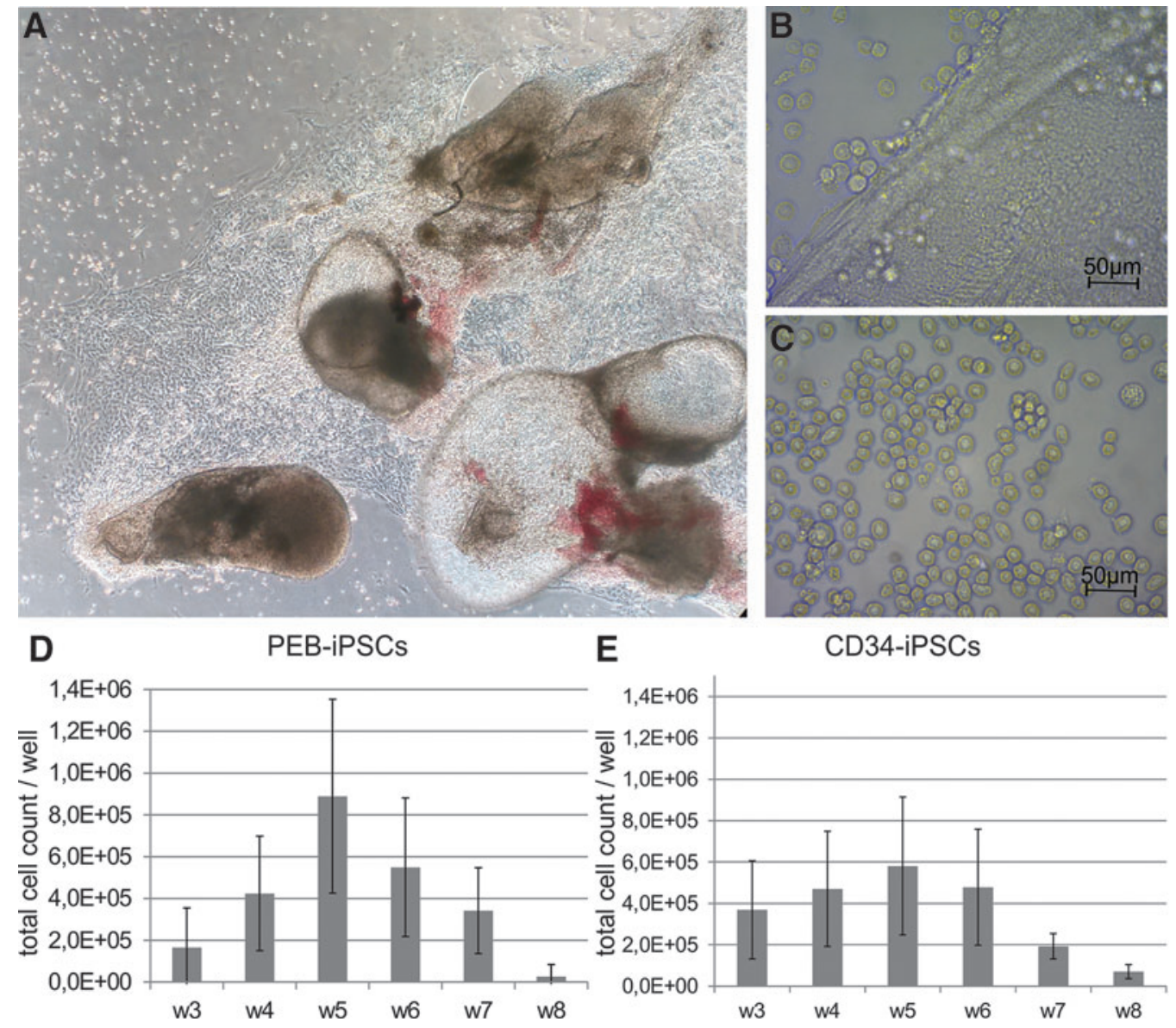

E

CD34-iPSCs

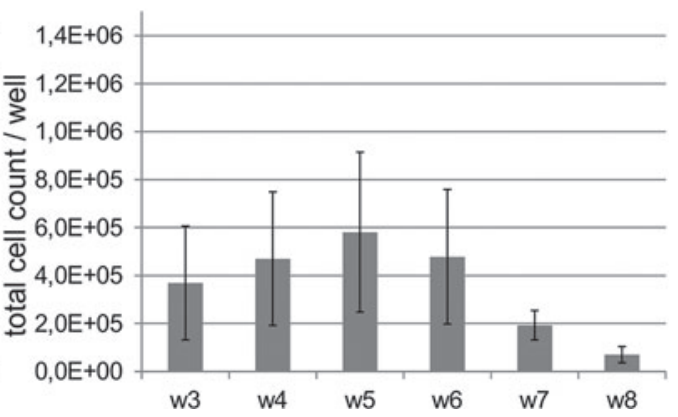

FIG. 2. Formation of an HCFC and release of hematopoietic cells into the supernatant. (A) Representative light microscopy image of the established HCFC, consisting of spheroids, red islands, and stromal cells and released cells (Primovert Zeiss; $4 \times$ ). (B) Stromal layer with single cells (scale bar $50 \mu \mathrm{m}$ ). (C) Disseminated single cells released into the supernatant (scale bar $50 \mu \mathrm{m})$. (D, E) Total number of cells released from the HCFC into the supernatant over the time course of 6 weeks (weeks 3-8 of the hematopoietic induction phase). Given are the absolute cell counts (mean \pm SD) for one well of a six-well plate per week for PEB-iPSCs $(n=5)(\mathbf{D})$ and CD34-iPSCs $(n=5)(\mathbf{E})$. SD, standard deviation. 


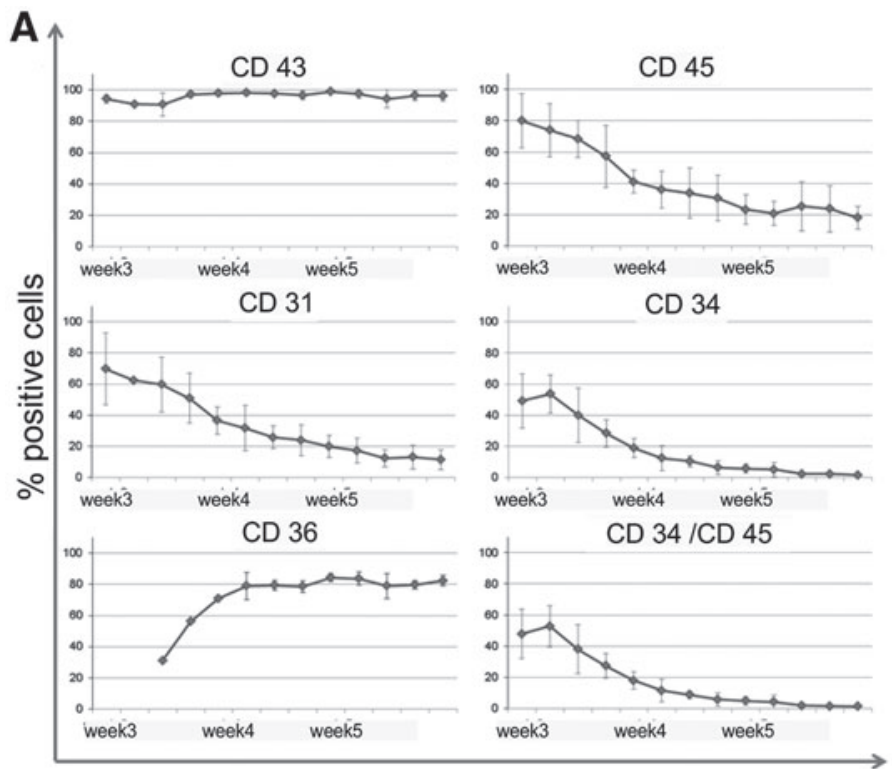

weeks of hematopoietic induction

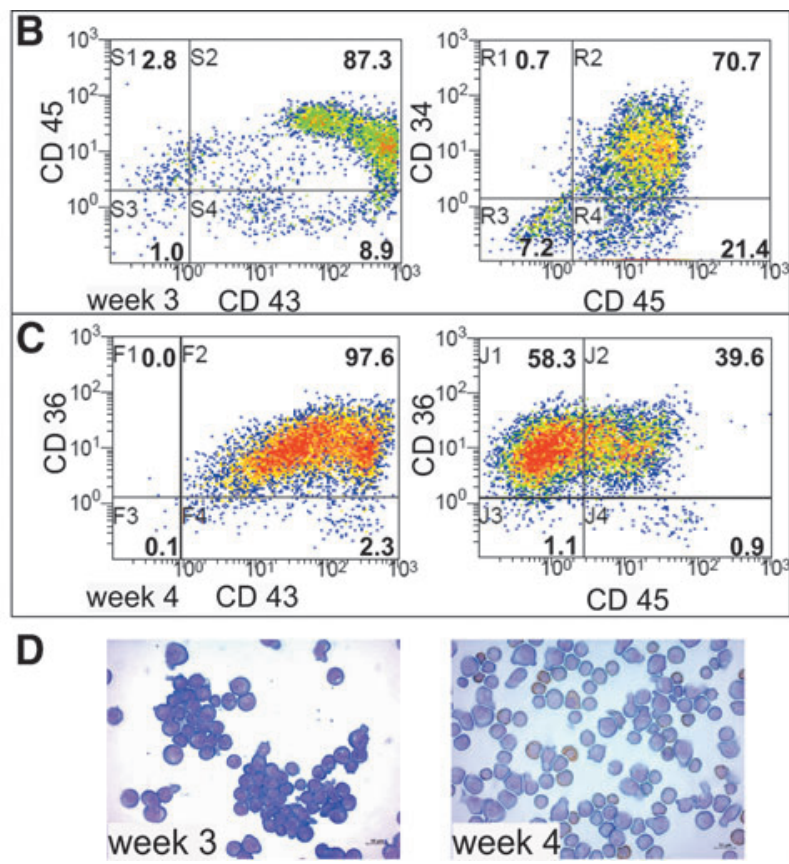

FIG. 3. Hematopoietic specification of cells released from the HCFC. Characterization of cells harvested on an almost daily basis from the supernatant (weeks 3-5 of hematopoietic induction). (A) Kinetics of the expression of cell surface markers (mean $\pm \mathrm{SD}$ ) measured by flow cytometry (CD34-iPSCs, $n=3$ ). (B, C) Representative flow cytometry analyses of cells from week 3 (B) for CD43, CD34, and CD45 and from week 4 (C) for CD43, CD45, and CD36. (D) Morphology of cells released into the supernatant at the beginning of weeks 3 and 4 and stained with MGG and neutral benzidine (scale bar: $10 \mu \mathrm{m})$. MGG, May-Grünwald Giemsa.

structures could not be avoided, wells containing only these structures were discarded.

\section{Characterization of single cells released into the supernatant}

The hematopoietic commitment of cells released from the HCFC into the supernatant was analyzed by flow cytometry, microscopy, and colony formation assays in semisolid media (Figs. 3 and 4). For flow cytometry analysis of cell surface marker expression, cells within the supernatant were harvested almost daily (Fig. 3A). As indicated by CD43 expression [23], the released cells were predominantly of a hematopoietic nature $(>90 \%)$. This selective release of $\mathrm{CD} 43^{+}$hematopoietic cells remained stable over the entire culture period. The majority of initially released cells (week
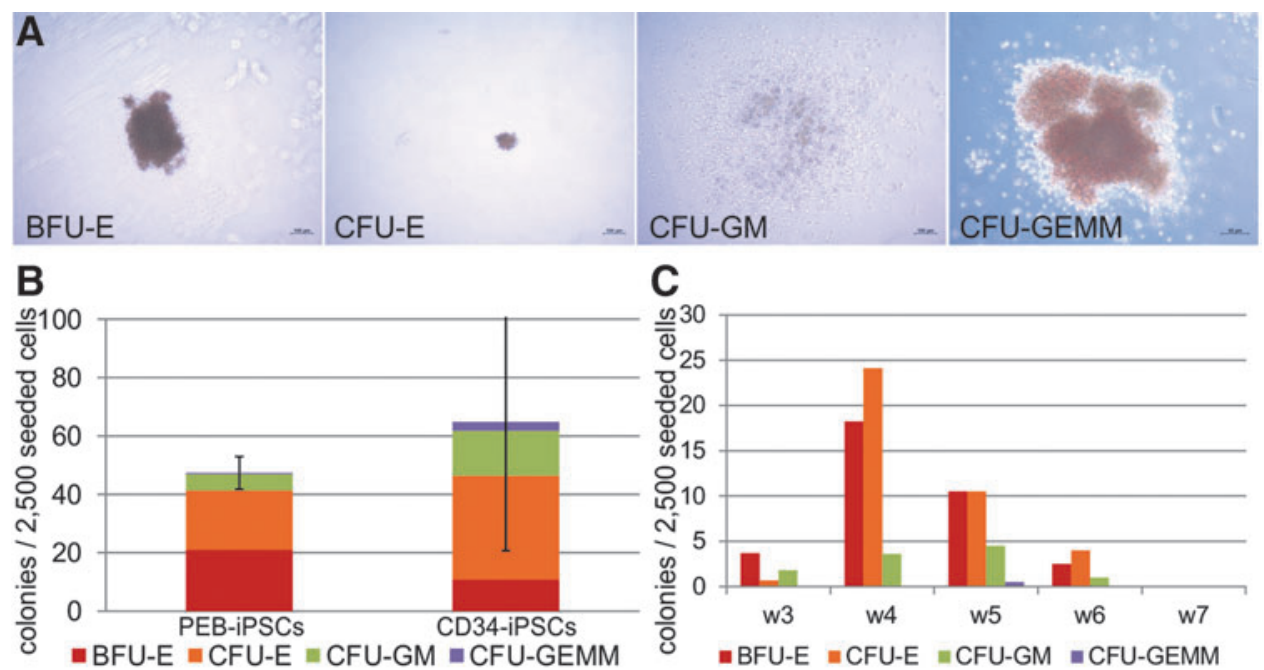

FIG. 4. Hematopoietic colony formation of cells released from the HCFC. (A) Representative light microscopy images of colony types (scale bar: $100 \mu \mathrm{m}$ ). (B) Hematopoietic colony formation in semisolid media per 2500 seeded cells from week 4 of hematopoietic induction (PEB-iPSCs, $n=4$; CD34-iPSCs, $n=6$; mean total colonies \pm SD). (C) Time course for colony formation during the entire hematopoietic induction period. Cells were harvested weekly (weeks 3-7) from the supernatant of PEB-iPSCderived HCFC for enumeration of their colony-forming potential. Shown are data from one representative experiment. 
$3)$ expressed the hematopoietic marker CD45 $(80 \% \pm 7.4 \%)$ together with CD34 $(52.8 \% \pm 13.1 \%)$, consistent with the phenotypic characteristics of hematopoietic stem/progenitor cells (HSPCs) (Fig. 3B). Morphologically, these cells resembled immature blasts with basophilic cytoplasm (Fig. 3D). During further EB cultivation, the percentage of $\mathrm{CD}_{3} 4^{+}$/ $\mathrm{CD} 45^{+}$cells in the supernatant decreased rapidly. $\mathrm{Si}-$ multaneously, the cells continuously changed phenotypically into $\mathrm{CD} 36^{+} / 45^{+}$early erythroblasts (week 4) and later (week 5) into $\mathrm{CD} 6^{+} / \mathrm{CD} 45^{-}$erythroblasts showing morphological characteristics of basophilic and subsequently hemoglobin-positive erythroblasts (Fig. 3C, D). Notably, $>80 \%$ of cells during the 4 th week were $\mathrm{CD}^{+} 6^{+}$and were therefore erythroid committed. Additional staining of GPA demonstrated that some of the $\mathrm{CD}^{+} 6^{+}$cells already expressed GPA. However, minimal cell numbers did not allow for continuous monitoring of GPA.

We further investigated the generation of proliferative progenitor stages by assessing colony formation in semisolid media (Fig. 4). Harvested cells (week 4) from all hiPSC lines gave rise to hematopoietic colonies with a high percentage of burstforming unit-erythroid (BFU-E) and colony-forming uniterythroid (CFU-E) cells (Fig. 4A, B). CD34-iPSCs showed the highest colony-forming potential with 1 colony per 39 seeded cells. The colony-forming potential of PEB-iPSCs, which was analyzed in more detail, decreased over time, consistent with the observed phenotypic changes and maturation of cells extruded from the HCFC (Fig. 4C). These results demonstrate a homogeneous and synchronous induction of hematopoiesis and erythropoiesis under the described culture conditions.

\section{Erythroid differentiation of cells released from the HCFC}

Next, we carefully evaluated the complete erythroid differentiation potential of hematopoietic cells released from the HCFC. For this purpose, cells obtained from the culture supernatant were cultivated in an established three-phase erythropoiesis system over 18 days [17]. The phenotypic characteristics of the harvested cells (week 4) from both iPSC sources used for ex vivo erythropoiesis are summarized in Supplementary Fig. S3. After 8 days of cultivation in EPO differentiation medium, the major fraction included erythroid precursors, as determined by the cell surface expression of CD36 (>90\%) and GPA (96.0\% $\pm 4 \%)$. Microscopic evaluation demonstrated a predominance of basophilic $(27.3 \% \pm 13.3 \%)$ and polychromatic $(62.1 \% \pm 9.1 \%)$ erythroblasts. During further differentiation, characteristic reductions in cell diameter and granules were observed along with gradually augmented hemoglobin content. On the final culture days, all cells were $\mathrm{GPA}^{+}$and band $3^{+}(>99 \%)$. Consistent with terminal maturation, CD36 expression decreased $(63.5 \% \pm 9.3 \%)$ and CD45 and $\alpha 4$-integrin (CD49d) expression disappeared [24]. The results for the erythroid differentiation of PEB-iPSCs are presented in Fig. 5A-C and Supplementary Fig. S4. Comparable differentiation was observed for CD34-iPSCs (Supplementary Figs. S4 and S5). Morphological analysis revealed that all cells were hemoglobin positive. Hemoglobin analyses at the protein level by high performance liquid chromatography (HPLC) (after $>15$ days of erythropoiesis) revealed the presence of predominantly fetal hemoglobin in hiPSC-derived RBCs, with minor contributions of embryonic and adult hemoglobin
(Fig. 5E). This hemoglobin pattern remained stable over time and was not dependent on the week of $\mathrm{CD} 43^{+}$cell harvest for further erythroid differentiation (Supplementary Fig. S6). In PEB-iPSC-derived cRBCs, an average of $38.6 \% \pm 17 \%$ enucleated cells was observed. Comparable enucleation was achieved in CD34-iPSCs $(37.1 \% \pm 4 \%)$. Notably, the maximum enucleation rate in PEB-iPSC-originating erythroid cells was $60 \%$ (Fig. 5B and Supplementary Fig. S6). The proliferation maximum was reached on day 15 . The mean cumulative expansion for PEB-iPSCs was 800-fold, as calculated from the number of $\mathrm{CD}_{4} 3^{+}$cells seeded on day 0 (Fig. 5D). Sufficient harvesting of cells for further erythropoiesis was feasible for 6 weeks, on average, between the third and eighth week of hematopoietic induction.

\section{Impact of cell interactions on enucleation}

Compared to other published methods, this method yielded a relatively high level of observable enucleation $(\sim 40 \%)$ during erythropoiesis from both CD34-iPSCs and PEBiPSCs. To affirm that the high enucleation rates observed for the newly generated PEB-iPSCs were caused by the adhesion culture conditions rather than cell line-specific factors, PEBiPSCs were cultured in this HCFC-based system and in our earlier described standard EB-based suspension culture [17] in parallel $(n=6)$ (Fig. 6A). In the latter, single cells derived from EBs digested after 21 days of cytokine stimulation in suspension were differentiated by using the same three-phase erythropoiesis system described for this study. Cells from the EB suspension showed homogeneous erythroid maturation into hemoglobin ${ }^{+}$cells at terminal maturation stages. Maturation resulted in $20.2 \% \pm 11.7 \%$ enucleation, which is consistent with the value in our previous report, but significantly lower than that for RBCs originating from PEB-iPSCs in this HCFC-based culture system $(44 \% \pm 15.1 \%, P<0.01)$ (Fig. 6B). Low enucleation of CD34-iPSCs (27\%) in our standard suspension system has already been reported [17]. One reason for the enhanced enucleation in this HCFC-based system might be an interaction of hematopoietic cells with other cells (eg, macrophages) or stromal elements. To confirm this hypothesis, we performed sorting experiments on $\mathrm{CD}_{4} 3^{+}$cells after EB digestion. In a set of five experiments (PEB-iPSCs, $n=2$; CD34-iPSCs, $n=3$ ), unsorted cells containing $>80 \%$ nonhematopoietic $\mathrm{CD}^{-} 3^{-}$cells and sorted $\mathrm{CD}_{4} 3^{+}$hematopoietic cells were subjected to the erythropoiesis system in parallel. Interestingly, unsorted cells underwent reaggregation into EB-like spheroids over 1 week and showed significantly higher enucleation rates $(34 \% \pm 17 \%)$ than $\mathrm{cRBCs}$ from the $\mathrm{CD} 43^{+}$sorted population $(15 \% \pm 7.8 \%$, $P \leq 0.05$ ) (Fig. 6C-E). These results confirm the necessity of cellular interactions for efficient erythroid differentiation.

\section{Characterization of the HCFC}

To gain more insight into the composition of the HCFC, we performed digestion after 5 weeks of cultivation and characterization of single cells by flow cytometry $(n=6)$. At this time, stromal layers were fully formed and had the greatest potential for further characterization.

Compared to the predominance of $\mathrm{CD} 43^{+}$hematopoietic cells in the supernatant, digested HCFC showed less hematopoietic commitment $(32.1 \% \pm 20.3 \%)$. Most of the CD $43^{+}$ 

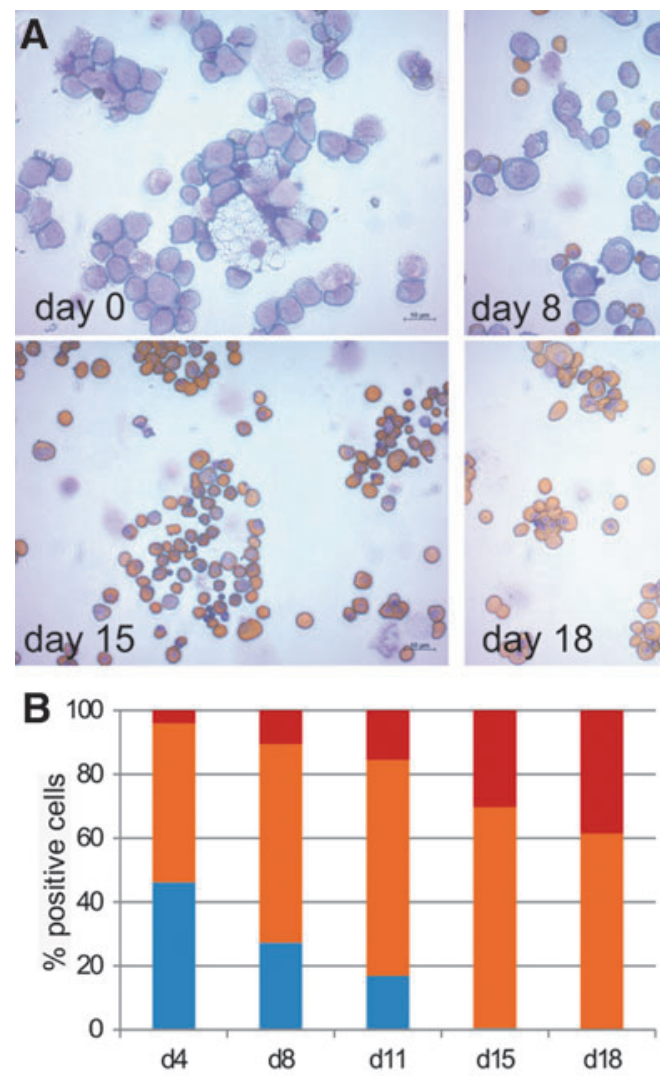

= basophilic erythroblasts = Hb+ erythroblasts $\approx$ reticulocytes

D

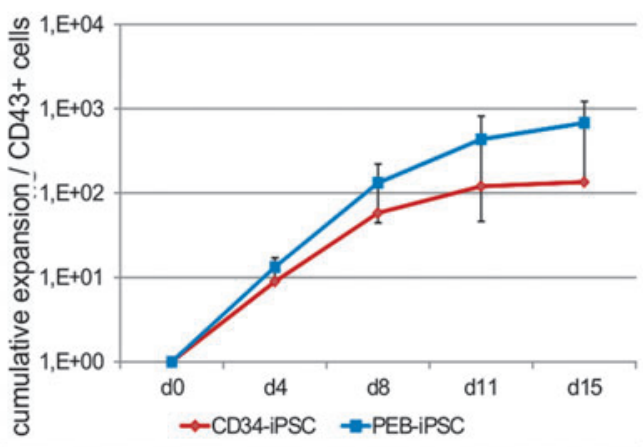

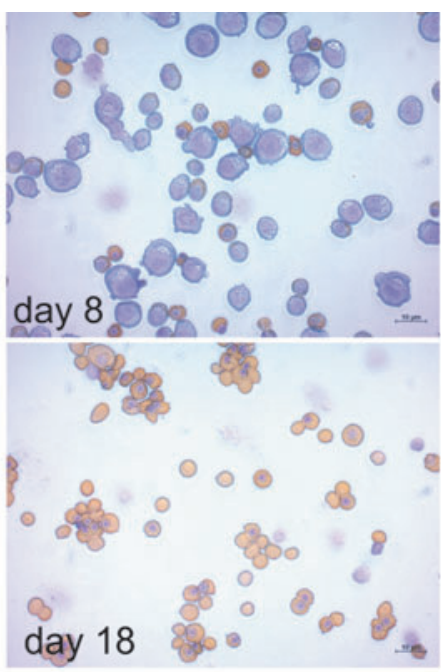
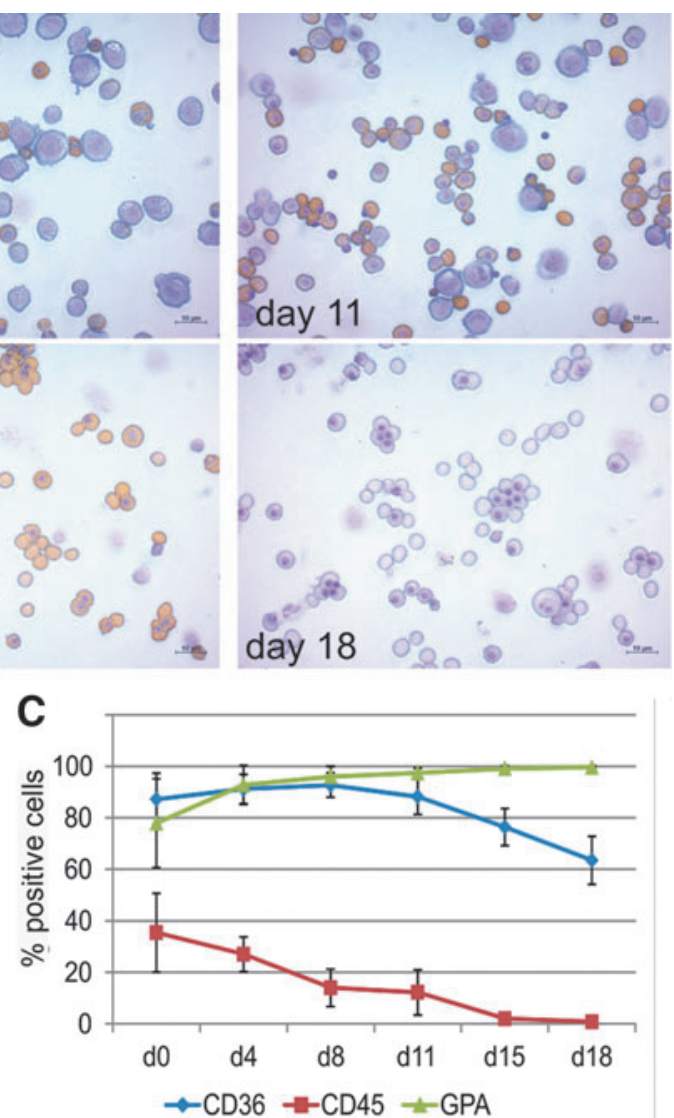

E

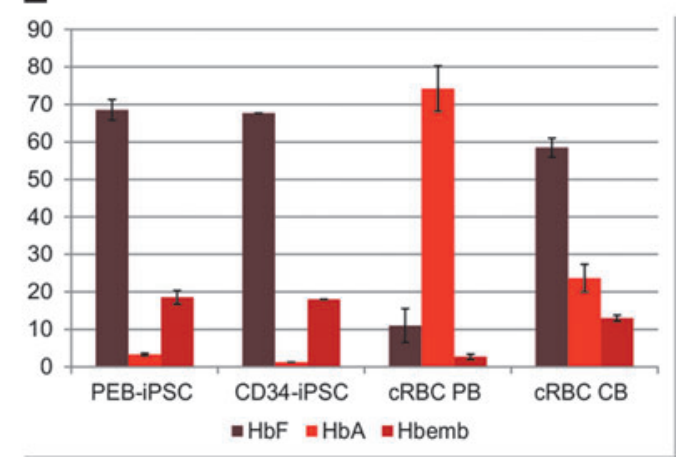

FIG. 5. Erythroid differentiation in a three-phase erythropoiesis system. (A) Representative images of cytospin samples derived from PEB-iPSCs costained with MGG and neutral benzidine. Day 18 cells were additionally stained with only MGG for a more valid evaluation of enucleation (scale bar: $10 \mu \mathrm{m}$ ). See also Supplementary Fig. S7 for confirmation of enucleation rates by Hoechst 33342 staining. (B) Differential counts of stained cytospin samples from days 4 to 18 (PEB-iPSCs, $n=6$ ). See also Supplementary Fig. S5 for CD34-iPSCs. (C) Flow cytometry analyses of cell surface marker expression in PEBiPSCs $(n=6$; mean \pm SD). See also Supplementary Fig. S4 for the expression of band 3 and CD49d and Supplementary Fig. S5 for CD34-iPSCs. (D) Cumulative expansion of erythroid cells from days 0 to 15 (PEB-iPSCs, $n=6$; CD34-iPSCs, $n=4$; mean \pm SD). (E) Hemoglobin composition of cRBCs as measured by HPLC. cRBCs from peripheral blood (cRBC PB, $n=5$ ) and cord blood (cRBC CB, $n=2$ ) $\mathrm{CD} 34^{+}$cells were used as controls (mean $\pm \mathrm{SD}$ ). See also Supplementary Fig. S6 for hemoglobin composition over time. HPLC, high-performance liquid chromatography; cRBCs, cultured RBCs.

cells were $\mathrm{CD}^{+} 5^{+}$erythroid and myeloid blasts (Supplementary Fig. S8A). Morphological analysis of cytospin preparations further demonstrated the presence of mature macrophages (Supplementary Fig. S8D). Flow cytometry analysis confirmed the presence of $10.5 \% \pm 7.9 \% \mathrm{CD} 86^{+}$cells

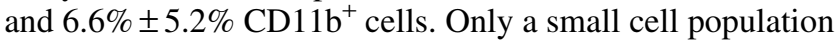
expressed CD14 $(2.5 \% \pm 1.2 \%)$ or CD163 $(2.4 \% \pm 1.2 \%)$, (Supplementary Fig. S8B).
Regarding the nonhematopoietic compartment, the expression of the endothelial markers VE-cadherin (CD144) and VEGF-R2 (CD309) was only marginal (CD144: $<1.5 \%$; CD309: $<2.5 \%)$. In contrast, a remarkable $\mathrm{CD} 31^{+}$population was detectable $(13.1 \% \pm 8.8 \%)$ (Supplementary Fig. S8C). Notably, all $\mathrm{CD} 31^{+}$cells were $\mathrm{CD} 43^{\text {high }}$ and $\mathrm{CD} 45^{+}$, and therefore of hematopoietic rather than endothelial origin (Supplementary Fig. S9A) [23]. This was confirmed by sorting experiments. 
A

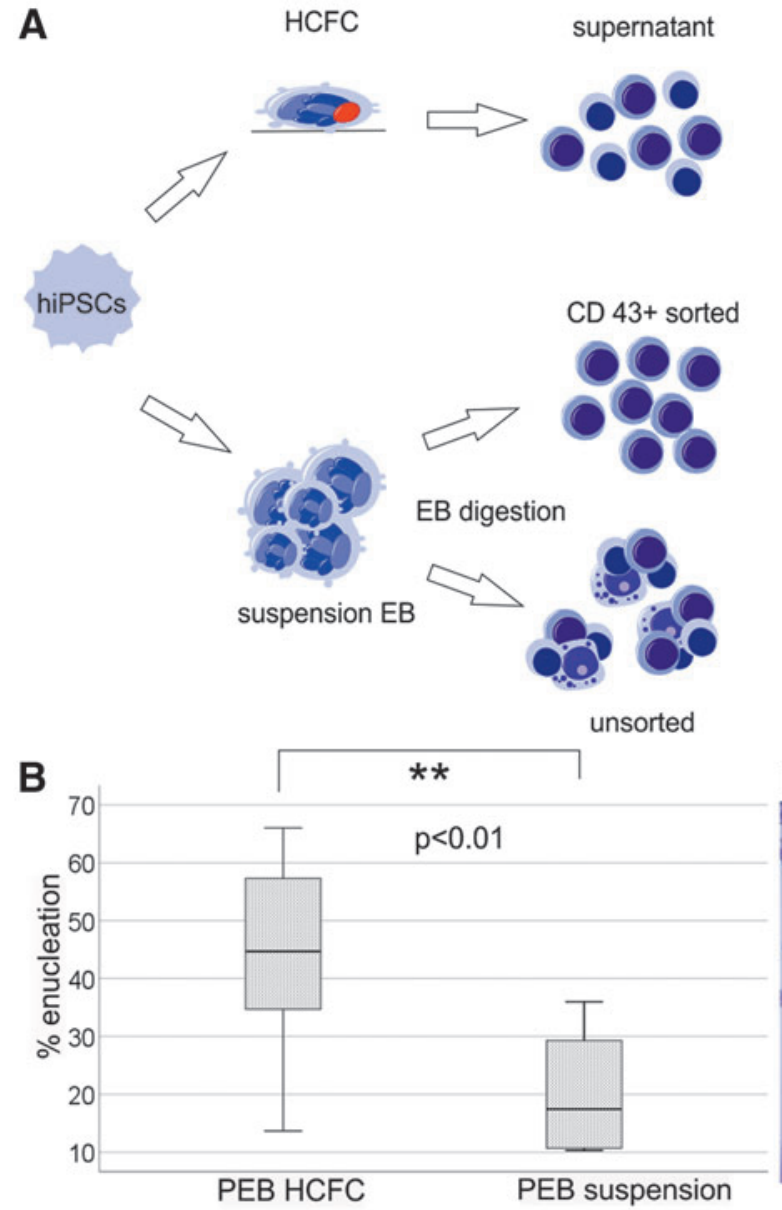

HCFC
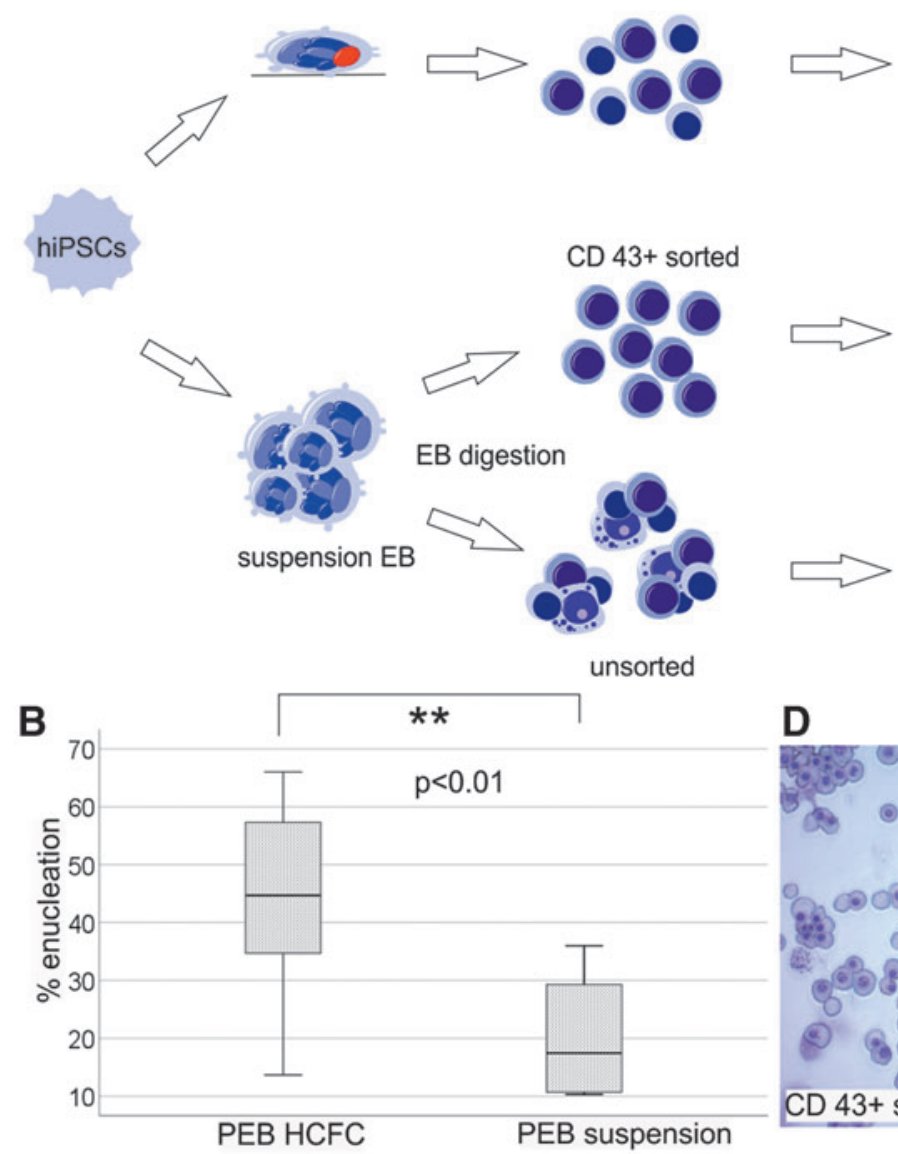

erythropoiesis

C
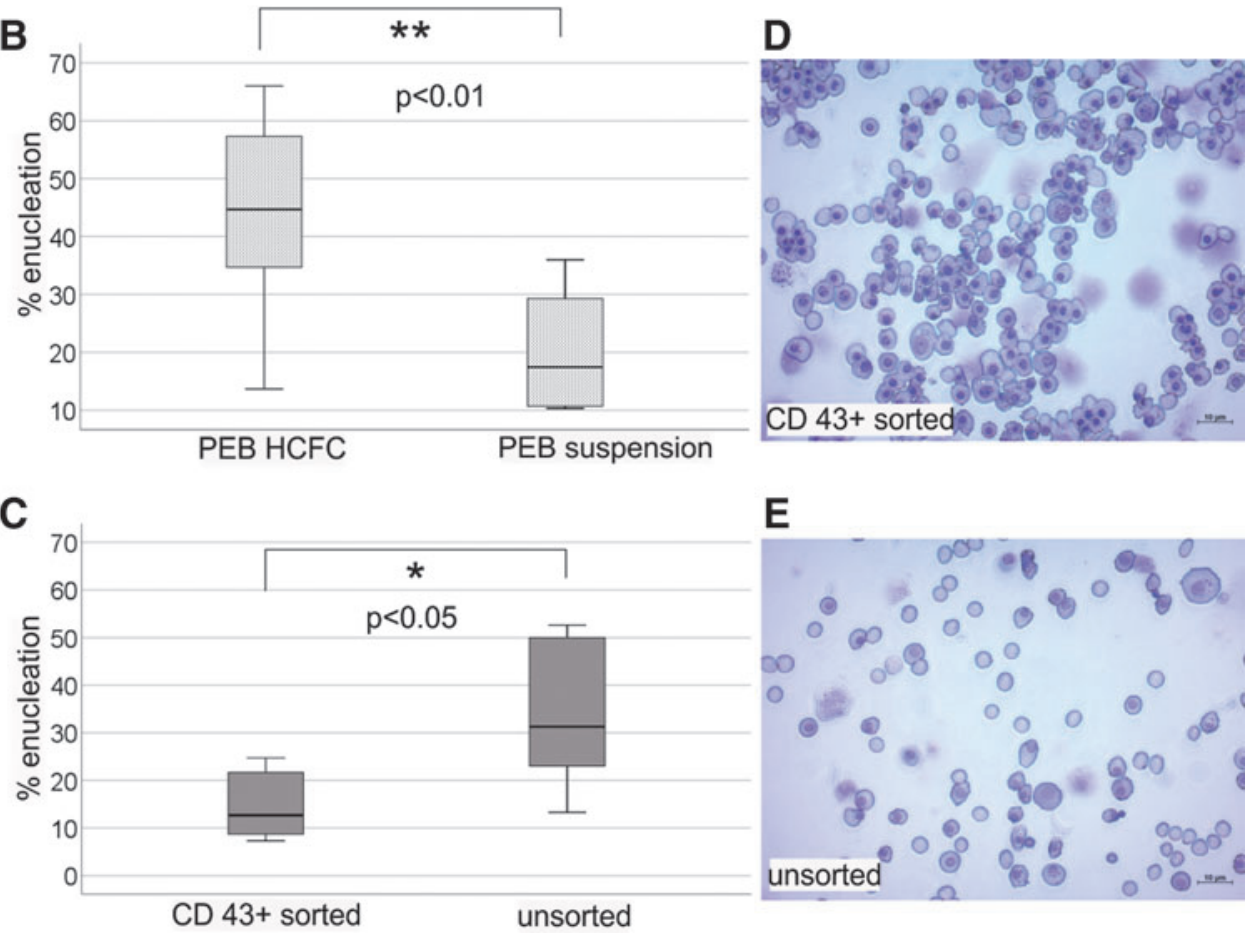

E

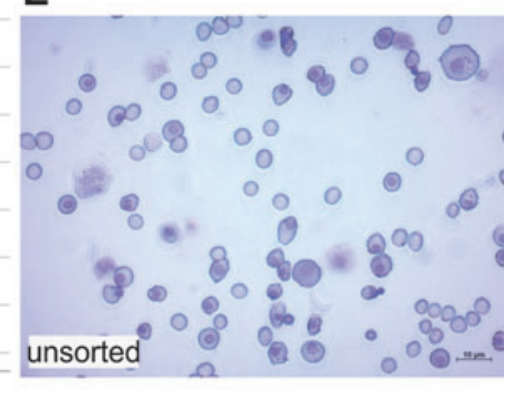

FIG. 6. Cellular interaction and enucleation. (A) Schematic drawing of the experimental setup comparing current HCFC-based and conventional suspension EB systems with and without sorting of $\mathrm{CD} 43^{+}$cells before erythroid differentiation. (B) Comparison of the enucleation rates of PEB-iPSCs cultured with this protocol (PEB HCFC, $n=6$ ) and the conventional suspension EB protocol (PEB suspension, $n=6)$. The plots show the median \pm 5 th $/ 95$ th percentiles $(* * P<0.01)$. (C) Enucleation rates of erythropoiesis cultures generated in parallel from $\mathrm{CD} 43^{+}$sorted and unsorted cells from the same source of iPSCs $(n=5, * P<$ $0.05 ;$ median \pm 5 th $/ 95$ th percentiles). (D, E) Representative images of MGGstained cytospin samples showing enucleated cRBCs in erythropoiesis culture from $\mathrm{CD}_{4} 3^{+}$sorted compared to unsorted cells (scale bar: $10 \mu \mathrm{m})$.
Sorted subpopulations of $\mathrm{CD} 31^{+}$cells showed high hematopoietic colony formation (Supplementary Fig. S9B, D), but no distinct net formation on ECMatrix compared to HUVECs (positive control) (Supplementary Fig. S9C). Several other cell types of uncertain origin could be detected after EB digestion, and these cells and their interactions are the subject of ongoing research (Supplementary Fig. S10).

\section{Discussion}

Hematopoiesis is a complex process involving carefully balanced interactions among cytokines, different cell types, matrix factors, and signaling pathways $[1,8,11]$. Due to ethical reasons and the inaccessibility of embryonic material, the molecular mechanisms driving hematopoiesis during early human development are largely unknown. As a result, appro- priate ex vivo systems for hiPSCs modeling the physiological steps of erythropoiesis are still challenging, and established culture systems are inefficient in terms of expansion and terminal differentiation [2,3]. Based on our recent observations for large-scale granulocyte and macrophage generation from hiPSCs using low cytokine support (IL-3, M-CSF/G-CSF) [21], we created a simplified erythropoiesis model.

For the initiation of hematopoietic development, EBs stimulated with only SCF, EPO, and IL-3 were allowed to form adherent 3D spheroids with stroma-like layers, termed "hematopoietic cell forming complex." The growth factors EPO and SCF are well known to be crucial for human definitive erythropoiesis, at least under ex vivo conditions [25-27]. Furthermore, the expression of the SCF receptor has been described within intra-aortic hematopoietic clusters as well as on the first HSCs that emerge in the human embryo [28,29]. 
A supportive effect of IL-3 on HSC development and EPOdependent erythropoiesis has been described in mice and humans [30,31]. In our model, both stromal layer formation and the shaping of vesicular spheroids were identified as being crucial for the formation of an HCFC and further hematopoietic differentiation. After $\sim 2$ weeks, red islands became detectable inside the fully formed HCFC. Hematopoietic cells were continuously released into the supernatant over a period of 6 weeks and could be harvested repeatedly for further erythroid differentiation. The majority $(>90 \%)$ of the released cells were hematopoietic and erythroid in particular, rendering further purification steps unnecessary.

To our knowledge, sufficient generation of hematopoietic/ erythroid progenitors has not been described using other protocols established for hiPSC-derived erythropoiesis. Only Ye et al. explicitly described high yields of progenitors from polycythemia vera-derived iPSCs [20]. In contrast to these previous observations, a remarkable population of $\mathrm{CD}^{+} /$ $\mathrm{CD} 45^{+}$hematopoietic and $\mathrm{CD} 45^{+} / \mathrm{CD} 36^{+}$erythroid progenitors was observed in this study, and the cells released from the HCFC showed high colony formation capacity (1/50) with a high percentage of erythroid colonies. For comparison, the colony formation described for BM mononuclear cells is $1 / 130$, and that described for purified BM CD34 ${ }^{+}$cells is 1/10 (HSPCs; STEMCELL Technologies). These results demonstrate a homogeneous and synchronous induction of hematopoiesis and erythropoiesis under the described culture conditions in which all physiological stages of human erythropoiesis were displayed, including proliferative erythroid progenitor stages.

Due to the continuous maturation of hematopoietic cells inside the HCFC over 6 weeks, a total of $1.5 \times 10^{7}$ erythroid progenitor/precursor cells could be harvested from one six-well plate. Therefore, a total volume of $150 \mathrm{~mL}$ of medium with cytokines was consumed. Further erythroid differentiation resulted in additional 100- to 1000-fold amplification. This corresponds to the generation of 34,400 erythroid cells from 1 hiPSC. Higher RBC yields have been reported by Olivier et al. [19] and Dias et al. [13]. Compared to our approach, the protocol of Olivier et al. is more complex, as it uses $>12$ growth factors in different combinations, and the protocol of Dias et al. uses xenogenic feeder cells. Most importantly, both prior protocols were limited by low enucleation rates below $10 \%$. Very recently, Olivier et al. proceeded in the development of a chemically defined albumin-free and low-transferrin erythroid differentiation protocol allowing for high expansion and comparable high enucleation (40\%). These advantages could be achieved using a relatively complex protocol with $>15$ cytokines/supplements during seven different culture phases [32].

In our system, the released hematopoietic cells showed homogeneous differentiation into hemoglobin-producing erythroid cells at terminal differentiation stages. Hemoglobin analyses on the protein level demonstrated predominantly fetal hemoglobin, indicating rather definitive erythropoiesis; this erythropoiesis was most likely comparable to fetal liver erythropoiesis, since the switch to adult hemoglobin was marginal. Compared to other established systems in the field, our novel approach yielded enhanced enucleation rates (mean 40\%, up to $60 \%$ ). Until now, published enucleation rates have been between $10 \%$ and $20 \%[12,13,18,19]$. Although we cannot completely exclude a cell line-specific [33] or epigenetic influence [34,35], another explanation for the enhanced enucleation in HCFC system may be an interaction between hematopoietic cells and other cells, for example, macrophages and stromal elements, inside the HCFC. The lack of such microenvironmental factors might explain the significantly lower fractions of already enucleated reticulocytes in conventional suspension cultures.

In support of this theory, culturing PEB-iPSCs and CD34iPSCs in our standard EB suspension culture resulted in only $20 \%$ and $27 \%$ enucleation, consistent with our previous report for other iPSC lines and significantly lower than observed with the new HCFC-based system [17]. This clearly demonstrates that the use of blood-derived iPSCs and their epigenetic signatures is not the only reason for enhanced enucleation from HCFC-derived cells [33-35]. In addition, after enzymatic dissociation of suspended EBs, the cells showed a high tendency to cluster into spheroid aggregates. After initial attempts to prevent this reaggregation, we realized that this side effect may positively influence erythroid maturation. Confirming this hypothesis, RBCs derived from sorted $\mathrm{CD}_{4} 3^{+}$hematopoietic cells after $\mathrm{EB}$ digestion (PEB-iPSCs and CD34-iPSCs) revealed significantly lower enucleation rates $(15 \%)$ than those derived from a mixture of unsorted cells (34\%). These results demonstrate the importance of cellular interactions, or at least the presence of soluble factors derived from nonhematopoietic cells, for the induction of sufficient enucleation. This importance has also been noted by others who have demonstrated the occurrence of enhanced proliferation/enucleation after coculture of erythroblasts with stromal cells or after injection of erythroblasts into the circulation of NOD-SCID mice [14,36,37].

In human BM, RBCs mature in close contact with macrophages in so-called erythroblastic islands (EBIs). EBIs have also been described in the fetal liver and are believed to support erythroblast proliferation, differentiation, survival, and enucleation [38-40]. However, ex vivo cultures of adult CD34 ${ }^{+} \mathrm{HSCs}$ show $>85 \%$ enucleation in the absence of macrophages $[41,42]$. This observation calls into question the essential role of macrophages in erythroblast enucleation at terminal stages of erythropoiesis. Considering our results and recent observations by others [43], we rather hypothesize that cellular interactions are already necessary during early erythroid or even hematopoietic development. During the submission of this article, this was also hypothesized by Shen et al. [44]. These authors studied the influence of sequential cellular niches on the generation of enucleated erythrocytes. Using endothelial-like feeder cells followed by murine OP9 stromal cells, they achieved comparably high enucleation rates. They also concluded that a specific niche is required already at early stage to prime hemogenic endothelial cells to generate erythroblasts with full maturation, especially enucleation, potential. In addition, comparable observations have been reported by Takayama et al. for platelet generation from hESCs by so-called "ES-sacs" [45]. These ESsacs resemble macroscopically our HCFC as they are described as balloon-like structures that are enriched in hematopoietic progenitors with multilineage potential. These authors also postulated that ES-sacs are indispensable for efficient production of fully differentiated hematopoietic cells, including platelets, as they provide the necessary microenvironment. Like in our system, cultures that did not form ES-sacs failed to differentiate into hematopoietic cells. More recently, this ES-sac system has also been used to generate improved levels of definitive erythroid cells, although enucleation rates were not reported [14].

To identify such interactions in our system, we tried to characterize the cellular composition of the HCFC. Interestingly, we observed only few endothelial-like cells, controversial to what 


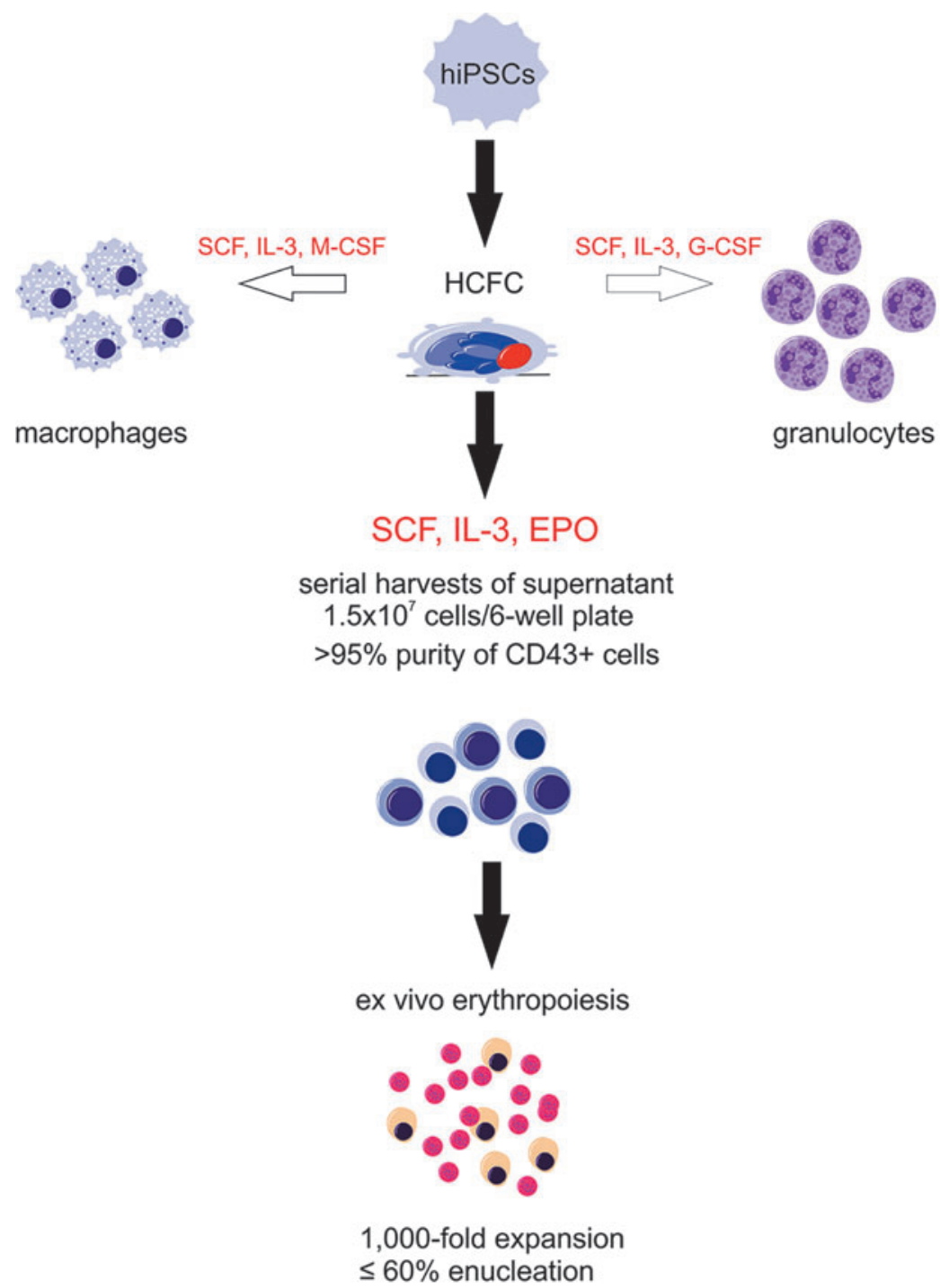

FIG. 7. Schematic overview illustrating the simplified xeno- and feeder free culture model for the ex vivo generation of RBCs from hiPSCs. High cellular output and enhanced enucleation are achieved using only three cytokines (EPO, IL-3, and SCF) and preserving the structure of the initially formed HCFC. The system may further be switched to granulocyte and macrophage generation by slight modification of added cytokines. could be presumed form the above-mentioned studies [44,45]. In addition to a variety of cells of uncertain origin, macrophages were observed, partially surrounded by erythroid cells, and therefore morphologically comparable to EBIs. In human BM, several macrophage subtypes have been described that have not yet been fully characterized. EBI macrophages express CD163, CD169, VCAM-1 (CD106), and CD206 [46,47]. A recent report described the abundant expression of $\mathrm{CD} 11 \mathrm{~b}$ by cells within mouse EBIs [40]. In our experiments, flow cytometry analysis after EB digestion revealed the presence of $\mathrm{CD}^{+} 6^{+}$cells and $\mathrm{CD} 1 \mathrm{~b}^{+}$myeloid cells, as well as a low percentage of $\mathrm{CD}_{163}{ }^{+}$cells. However, there is increasing evidence that the phenotypes of macrophages supporting human hematopoiesis show high plasticity and are strongly influenced by both the microenvironment and cytokine support [48].

Overall, we established an improved and simplified xenoand feeder-free cell culture system for erythroid differentiation of hiPSCs, which is illustrated in Fig. 7. Compared to other approaches, as summarized for our standard EB suspension protocol [17] in Supplementary Table S2, this system has the following important advantages: minimal handling time (with medium exchange only once a week); lower costs due to reduced cytokine support and reduced medium consumption in relationship to the erythroid output; continuous harvesting of hematopoietic cells over 6 weeks without any digestion or purification; homogeneous maturation through all stages of human erythropoiesis, including the progenitor cell stage; and improved yield of enucleated RBCs. In addition, we previously demonstrated that the addition of IL-3 in combination with MCSF/G-CSF drives hematopoietic differentiation toward macrophages and granulocytes, demonstrating the flexibility of the system [21]. These advantages may be caused by improved in vitro mimicking of the physiological niche in which hematopoietic differentiation takes place due to the provision of tightly tuned cell-cell contacts and paracrine cytokine support in a formed HCFC. Therefore, this model represents a suitable system for studies on developmental biology and RBC generation. Studies are currently underway to characterize cellular interactions in the HCFC in more detail and to optimize the concept of an artificial niche for further improvement of RBC expansion from hiPSCs.

\section{Acknowledgments}

The authors would like to thank Marie-Therese Frisch and Anja Stoisser for their excellent technical assistance. 
The data discussed in this publication have been deposited in NCBI's Gene Expression Omnibus (GEO) [49] and are accessible through GEO Series accession number GSE125624 (www.ncbi.nlm.nih.gov/geo/query/acc.cgi\$acc=GSE125624).

\section{Author Disclosure Statement}

No competing financial interests exist.

\section{Funding Information}

The study was supported by research funding from the German Society for Transfusion Medicine and Immunohematology to I.D. and P.S., by a grant from the German Federal Ministry of Education and Research (BMBF 01GN1008B) to H.Z., and by grants DFG113/18 from the Diputación Foral de Gipuzkoa, Spain, Ministry of Economy and Competitiveness, Spain, MINECO grant BFU201677987-P and Instituto de Salud Carlos III (AC17/00012) cofunded by the European Union (Eraco-sysmed/H2020 Grant Agreement No. 643271) to M.J.A.-B.

\section{Supplementary Material}

Supplementary Data

Supplementary Figure S1

Supplementary Figure S2

Supplementary Figure S3

Supplementary Figure S4

Supplementary Figure S5

Supplementary Figure S6

Supplementary Figure S7

Supplementary Figure S8

Supplementary Figure S9

Supplementary Figure S10

Supplementary Table S1

Supplementary Table S2

\section{References}

1. Ackermann M, S Liebhaber, JH Klusmann and N Lachmann. (2015). Lost in translation: pluripotent stem cellderived hematopoiesis. EMBO Mol Med 7:1388-1402.

2. Focosi D and G Amabile. (2017). Induced pluripotent stem cell-derived red blood cells and platelet concentrates: from bench to bedside. Cells 7:1-10.

3. Wahlster L and GQ Daley. (2016). Progress towards generation of human haematopoietic stem cells. Nat Cell Biol 18:1111-1117.

4. Qiu C, EN Olivier, M Velho and EE Bouhassira. (2008). Globin switches in yolk sac-like primitive and fetal-like definitive red blood cells produced from human embryonic stem cells. Blood 111:2400-2408.

5. Van Handel B, SL Prashad, N Hassanzadeh-Kiabi, A Huang, M Magnusson, B Atanassova, A Chen, EI Hamalainen and HK Mikkola. (2010). The first trimester human placenta is a site for terminal maturation of primitive erythroid cells. Blood 116:3321-3330.

6. Migliaccio G, AR Migliaccio, S Petti, F Mavilio, G Russo, D Lazzaro, U Testa, M Marinucci and C Peschle. (1986). Human embryonic hemopoiesis. Kinetics of progenitors and precursors underlying the yolk sac-liver transition. J Clin Invest 78:51-60.

7. Palis J. (2014). Primitive and definitive erythropoiesis in mammals. Front Physiol 5:3.
8. Ditadi A, CM Sturgeon and G Keller. (2017). A view of human haematopoietic development from the Petri dish. Nat Rev Mol Cell Biol 18:56-67.

9. Nandakumar SK, JC Ulirsch and VG Sankaran. (2016). Advances in understanding erythropoiesis: evolving perspectives. Br J Haematol 173:206-218.

10. Arai F, A Hirao, M Ohmura, H Sato, S Matsuoka, K Takubo, K Ito, GY Koh and T Suda. (2004). Tie2/angiopoietin-1 signaling regulates hematopoietic stem cell quiescence in the bone marrow niche. Cell 118:149-161.

11. Ivanovs A, S Rybtsov, ES Ng, EG Stanley, AG Elefanty and A Medvinsky. (2017). Human haematopoietic stem cell development: from the embryo to the dish. Development 144:2323-2337.

12. Chang CJ, K Mitra, M Koya, M Velho, R Desprat, J Lenz and EE Bouhassira. (2011). Production of embryonic and fetal-like red blood cells from human induced pluripotent stem cells. PLoS One 6:e25761.

13. Dias J, M Gumenyuk, H Kang, M Vodyanik, J Yu, JA Thomson and II Slukvin. (2011). Generation of red blood cells from human induced pluripotent stem cells. Stem Cells Dev 20:1639-1647.

14. Fujita A, N Uchida, JJ Haro-Mora, T Winkler and J Tisdale. (2016). beta-Globin-expressing definitive erythroid progenitor cells generated from embryonic and induced pluripotent stem cell-derived sacs. Stem Cells 34:1541-1552.

15. Mao B, S Huang, X Lu, W Sun, Y Zhou, X Pan, J Yu, M Lai, B Chen, et al. (2016). Early development of definitive erythroblasts from human pluripotent stem cells defined by expression of glycophorin A/CD235a, CD34, and CD36. Stem Cell Reports 7:869-883.

16. Yang CT, A French, PA Goh, A Pagnamenta, S Mettananda, J Taylor, S Knight, A Nathwani, DJ Roberts, SM Watt and L Carpenter. (2014). Human induced pluripotent stem cell derived erythroblasts can undergo definitive erythropoiesis and co-express gamma and beta globins. Br J Haematol 166:435-448.

17. Dorn I, K Klich, MJ Arauzo-Bravo, M Radstaak, S Santourlidis, F Ghanjati, TF Radke, OE Psathaki, G Hargus, et al. (2015). Erythroid differentiation of human induced pluripotent stem cells is independent of donor cell type of origin. Haematologica 100:32-41.

18. Lapillonne H, L Kobari, C Mazurier, P Tropel, MC Giarratana, I Zanella-Cleon, L Kiger, M Wattenhofer-Donze, $\mathrm{H}$ Puccio, et al. (2010). Red blood cell generation from human induced pluripotent stem cells: perspectives for transfusion medicine. Haematologica 95:1651-1659.

19. Olivier EN, L Marenah, A McCahill, A Condie, S Cowan and JC Mountford. (2016). High-efficiency serum-free feeder-free erythroid differentiation of human pluripotent stem cells using small molecules. Stem Cells Transl Med 5:1394-1405.

20. Ye Z, CF Liu, L Lanikova, SN Dowey, C He, X Huang, RA Brodsky, JL Spivak, JT Prchal and L Cheng. (2014). Differential sensitivity to JAK inhibitory drugs by isogenic human erythroblasts and hematopoietic progenitors generated from patient-specific induced pluripotent stem cells. Stem Cells 32:269-278.

21. Lachmann N, M Ackermann, E Frenzel, S Liebhaber, S Brennig, C Happle, D Hoffmann, O Klimenkova, D Luttge, et al. (2015). Large-scale hematopoietic differentiation of human induced pluripotent stem cells provides granulocytes or macrophages for cell replacement therapies. Stem Cell Reports 4:282-296.

22. van Zwieten R, M Veldthuis, B Delzenne, J Berghuis, J Groen, F Ait Ichou, E Clifford, CL Harteveld and AK 
Stroobants. (2014). Hemoglobin analyses in the Netherlands reveal more than 80 different variants including six novel ones. Hemoglobin 38:1-7.

23. Vodyanik MA, JA Thomson and II Slukvin. (2006). Leukosialin (CD43) defines hematopoietic progenitors in human embryonic stem cell differentiation cultures. Blood 108:2095-2105.

24. Hu J, J Liu, F Xue, G Halverson, M Reid, A Guo, L Chen, A Raza, N Galili, et al. (2013). Isolation and functional characterization of human erythroblasts at distinct stages: implications for understanding of normal and disordered erythropoiesis in vivo. Blood 121:3246-3253.

25. Migliaccio G, AR Migliaccio, ML Druzin, PJ Giardina, KM Zsebo and JW Adamson. (1992). Long-term generation of colony-forming cells in liquid culture of $\mathrm{CD} 34^{+}$cord blood cells in the presence of recombinant human stem cell factor. Blood 79:2620-2627.

26. Muta K, SB Krantz, MC Bondurant and CH Dai. (1995). Stem cell factor retards differentiation of normal human erythroid progenitor cells while stimulating proliferation. Blood 86:572-580.

27. Wu H, U Klingmuller, P Besmer and HF Lodish. (1995). Interaction of the erythropoietin and stem-cell-factor receptors. Nature 377:242-246.

28. Ivanovs A, S Rybtsov, RA Anderson, ML Turner and A Medvinsky. (2014). Identification of the niche and phenotype of the first human hematopoietic stem cells. Stem Cell Reports 2:449-456.

29. Labastie MC, F Cortes, PH Romeo, C Dulac and B Peault. (1998). Molecular identity of hematopoietic precursor cells emerging in the human embryo. Blood 92:3624-3635.

30. Broxmeyer HE. (2013). Erythropoietin: multiple targets, actions, and modifying influences for biological and clinical consideration. J Exp Med 210:205-208.

31. Douay L, MC Giarratana, X Drouet, D Bardinet and NC Gorin. (1991). The role of recombinant haematopoietic growth factors in human long-term bone marrow culture in serum-free medium. Br J Haematol 79:27-32.

32. Olivier EN, S Zhang, Z Yan, S Suzuka, K Roberts, K Wang and EE Bouhassira. (2019). PSC-RED and MNC-RED: albumin-free and low-transferrin robust erythroid differentiation protocols to produce human enucleated red blood cells. Exp Hematol 75:31.e15-52.e15.

33. Nishizawa M, K Chonabayashi, M Nomura, A Tanaka, M Nakamura, A Inagaki, M Nishikawa, I Takei, A Oishi, et al. (2016). Epigenetic variation between human induced pluripotent stem cell lines is an indicator of differentiation capacity. Cell Stem Cell 19:341-354.

34. Kim K, R Zhao, A Doi, K Ng, J Unternaehrer, P Cahan, H Huo, YH Loh, MJ Aryee, et al. (2011). Donor cell type can influence the epigenome and differentiation potential of human induced pluripotent stem cells. Nat Biotechnol 29:1117-1119.

35. Lister R, M Pelizzola, YS Kida, RD Hawkins, JR Nery, G Hon, J Antosiewicz-Bourget, R O'Malley, R Castanon, et al. (2011). Hotspots of aberrant epigenomic reprogramming in human induced pluripotent stem cells. Nature 471:68-73.

36. Giarratana MC, L Kobari, H Lapillonne, D Chalmers, L Kiger, T Cynober, MC Marden, H Wajcman and L Douay. (2005). Ex vivo generation of fully mature human red blood cells from hematopoietic stem cells. Nat Biotechnol 23:69-74.

37. Neildez-Nguyen TM, H Wajcman, MC Marden, M Bensidhoum, V Moncollin, MC Giarratana, L Kobari, D Thierry and L Douay. (2002). Human erythroid cells produced ex vivo at large scale differentiate into red blood cells in vivo. Nat Biotechnol 20:467-472.
38. Chasis JA and N Mohandas. (2008). Erythroblastic islands: niches for erythropoiesis. Blood 112:470-478.

39. Manwani D and JJ Bieker. (2008). The erythroblastic island. Curr Top Dev Biol 82:23-53.

40. Seu KG, J Papoin, R Fessler, J Hom, G Huang, N Mohandas, L Blanc and TA Kalfa. (2017). Unraveling macrophage heterogeneity in erythroblastic islands. Front Immunol 8:1140.

41. Betz J, I Dorn, IU Kouzel, A Bauwens, I Meisen, B Kemper, M Bielaszewska, M Mormann, L Weymann, et al. (2016). Shiga toxin of enterohaemorrhagic Escherichia coli directly injures developing human erythrocytes. Cell Microbiol 18:1339-1348.

42. Giarratana MC, H Rouard, A Dumont, L Kiger, I Safeukui, PY Le Pennec, S Francois, G Trugnan, T Peyrard, et al. (2011). Proof of principle for transfusion of in vitrogenerated red blood cells. Blood 118:5071-5079.

43. Heideveld E, F Masiello, M Marra, F Esteghamat, N Yagci, $M$ von Lindern, AR Migliaccio and $E$ van den Akker. (2015). CD14 ${ }^{+}$cells from peripheral blood positively regulate hematopoietic stem and progenitor cell survival resulting in increased erythroid yield. Haematologica 100:1396-1406.

44. Shen J, Y Zhu, C Lyu, Z Feng, S Lyu, Y Zhao, DL Hoyle, G Ji, W Miao, et al. (2019). Sequential cellular niches control the generation of enucleated erythrocytes from human pluripotent stem cells. Haematologica [Epub ahead of print]; DOI: 10.3324/haematol.2018.211664.

45. Takayama N, H Nishikii, J Usui, H Tsukui, A Sawaguchi, T Hiroyama, K Eto and H Nakauchi. (2008). Generation of functional platelets from human embryonic stem cells in vitro via ES-sacs, VEGF-promoted structures that concentrate hematopoietic progenitors. Blood 111:5298-5306.

46. Chow A, M Huggins, J Ahmed, D Hashimoto, D Lucas, Y Kunisaki, S Pinho, M Leboeuf, C Noizat, et al. (2013). CD169(+) macrophages provide a niche promoting erythropoiesis under homeostasis and stress. Nat Med 19:429-436.

47. Fabriek BO, MM Polfliet, RP Vloet, RC van der Schors, AJ Ligtenberg, LK Weaver, C Geest, K Matsuno, SK Moestrup, CD Dijkstra and TK van den Berg. (2007). The macrophage CD163 surface glycoprotein is an erythroblast adhesion receptor. Blood 109:5223-5229.

48. Heideveld E, LA Hampton-O'Neil, SJ Cross, FPJ van Alphen, $\mathrm{M}$ van den Biggelaar, AM Toye and $\mathrm{E}$ van den Akker. (2018). Glucocorticoids induce differentiation of monocytes towards macrophages that share functional and phenotypical aspects with erythroblastic island macrophages. Haematologica 103:395-405.

49. Edgar R, M Domrachev and AE Lash. (2002). Gene Expression Omnibus: NCBI gene expression and hybridization array data repository. Nucleic Acids Res 30:207-210.

Address correspondence to: Isabel Dorn, MD

Department of Blood Group Serology and Transfusion Medicine Medical University Graz. 8036 Graz

Austria

E-mail: isabel.dorn@medunigraz.at

Received for publication June 26, 2019

Accepted after revision October 7, 2019

Prepublished on Liebert Instant Online October 9, 2019 